\title{
Modeling the photoreduction of amines by the triplet nitromethane
}

\author{
Dmitriy Ovsyannikov *, Dmitriy Fomichev, Sergei Zelentsov \\ Lobachevsky State University of Nizhni Novgorod \\ Correspondence: amidius.1992@gmail.com
}

\begin{abstract}
Photoreduction of ammonia, methylamine and phenylamine by the triplet nitromethane has been investigated. Using uB3LYP/6-311g* method the geometry of the transition states, pre- and post-complexes was established. The mechanism of photoreduction is quite simple: it is the hydrogen atom abstraction. The N-H-bond of amine breaks and $\mathrm{H}$-atom passes to the nitrocompound. This reaction yields two radicals. Calculated activation energy of the reaction between nitromethane and ammonia is of $3.17 \mathrm{kcal} \cdot \mathrm{mol}^{-1}$. This one is much lower comparing to the reported earlier by us. The EOM-CCSD/cc-pVDZ method predicts activation energy of $2.57 \mathrm{kcal} \cdot \mathrm{mol}^{-1}$. The next examined reaction is the reaction between the triplet nitromethane and methylamine. The mechanism is the same. Calculated activation energy is of $1.17 \mathrm{kcal} \cdot \mathrm{mol}^{-1}$. Due to the methyl group presence it is the energy drop relatively to the first reaction. Some pictures of the electron density are included in the article. Comparing the radicals the phenylamine with nitromethane has been implemented. Calculated activation energy is of $1.51 \mathrm{kcal} \cdot \mathrm{mol}^{-1}$. Hence phenyl group enhances the activation threshold of the proton abstraction. The reaction mechanism is the same hydrogen atom abstraction.
\end{abstract}

Keywords: photoreduction, amines, nitrocompound, excited state, hydrogen abstraction

\section{Introduction}

Photochemical hydrogen abstraction is one of the most interesting processes in photochemistry. It could be observed with the ketone-like structures having $\mathrm{C}=\mathrm{O}$ or $\mathrm{N}=\mathrm{O}$ fragments. One of the most important condition is a small singlet-triplet gap. The excitation must be proceeded via a $\left(\mathrm{n}, \pi^{*}\right)$ transition more than via a $\left(\pi, \pi^{*}\right)$-transition. The corresponding structures are, e.g., ketones, quinones and nitrocompounds. Present work takes into account excited nitrocompounds reacting with some of the amines. Amines could be a donor of the hydrogen atom.

\section{Computational details}

All DFT calculations were performed by NWChem [1] program. uB3LYP/6-311g* method was used. The Coupled Cluster [2] calculation were performed by PSI4 [3] program. Visualization of the molecules provided by Avogadro [4], [5] program using OpenBabel [6] code. Topology and electron density descriptors were calculated and visualized by Multiwfn [7] program. Graphs are builded by gnuplot program [8].

\section{Results and discussion}

The first reaction researched is hydrogen atom abstraction from monomethylamine by triplet nitromethane. This mechanism is important for many photochemical reactions [9], [10]. It was reported 
previously [11] that activation energy for this reaction has extremely high value of $370.8 \mathrm{~kJ} \cdot \mathrm{mol}^{-1}$. The reaction pathway represents abnormally plain profile of Potential Energy Surface (PES) with three minima's. But recalculated reaction pathway on the uB3LYP/6-311G* level of theory shows contradictory form. As we can see on fig. 1, new reaction pathway is a classical profile of PES with two minima's of pre- and postreaction complexes and shape of the Transition State (TS) peak. The activation energy is of $24.5 \mathrm{~kJ} \cdot \mathrm{mol}^{-1}\left(5.9 \mathrm{kcal} \cdot \mathrm{mol}^{-1}\right)$. The peak position on the fig. 1 looks like that activation energy should be above $25 \mathrm{~kJ} \cdot \mathrm{mol}^{-1}$, but the graph was smoothed by gnuplot program; barrier calculated by NEB method is of $24.5 \mathrm{~kJ} \cdot \mathrm{mol}^{-1}$. Using frequencies task, the Zero-Point Energycorrected (ZPE) value calculated as $13.3 \mathrm{~kJ} \cdot \mathrm{mol}^{-1}\left(3.2 \mathrm{kcal} \cdot \mathrm{mol}^{-1}\right)$.

The EOM-CCSD/cc-pVDZ calculations on the geometries obtained by DFT method were performed using PSI4 program . Due to some technical problems, we are not able to obtain ZPEcorrected energies. Calculated activation barrier is of $10.7 \mathrm{~kJ} \cdot \mathrm{mol}^{-1}\left(2.3 \mathrm{kcal} \cdot \mathrm{mol}^{-1}\right)$.

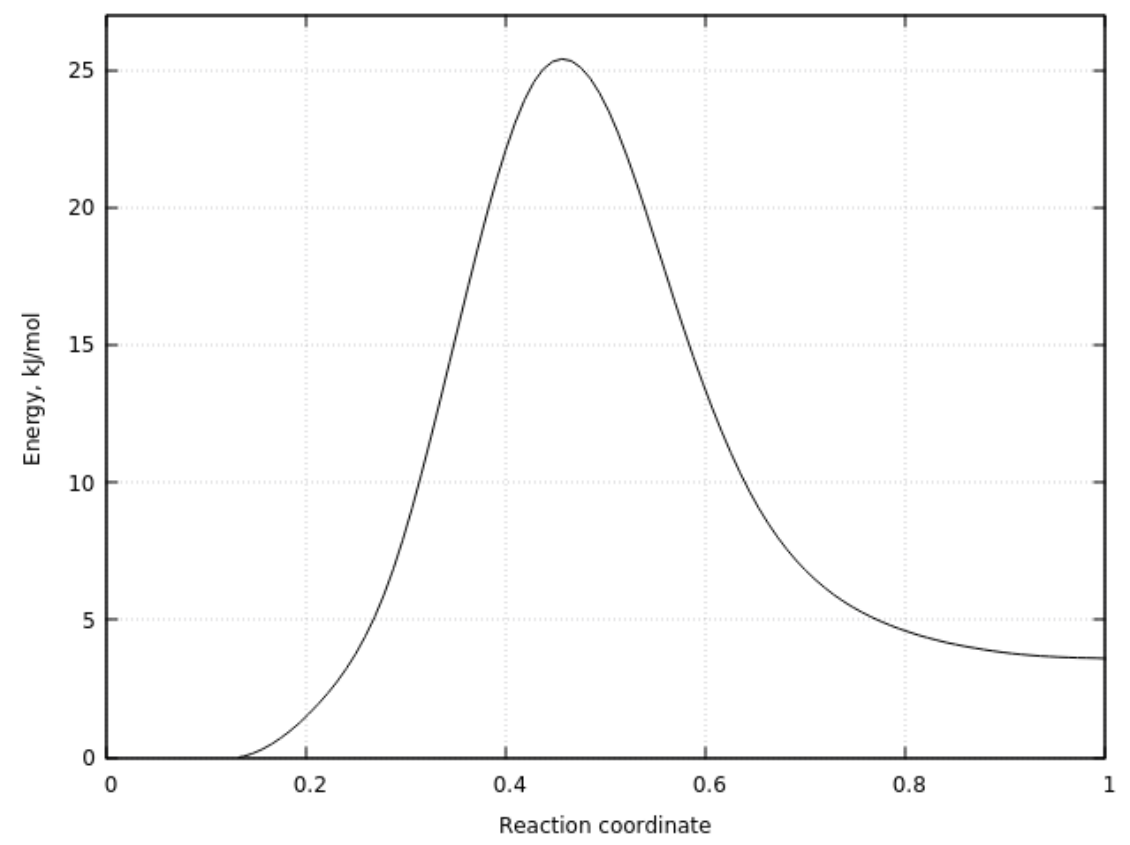

Figure 1: The reaction pathway between ammonia and nitromethane in the triplet state calculated using $u B 3 L Y P / 6-311 G^{*}$ method

The mechanism of the reaction is the photochemical hydrogen abstraction by the triplet nitromethane. On the fig. 2 we can see the transition state of the corresponding reaction. The enhanced $\mathrm{N}-\mathrm{H}$ bond length of $1.25 \AA$ of the ammonia corresponds to the reaction pathway. Let us note the geometry of the nitromethane. The $\mathrm{NO}_{2}$-group plane lays outside of the $\mathrm{C}-\mathrm{N}-\mathrm{O}$ plane. In the singlet state two planes $\mathrm{C}-\mathrm{N}-\mathrm{O}^{1}$ and $\mathrm{C}-\mathrm{N}-\mathrm{O}^{2}$ are coplanar. It raises the effect discussed below. 


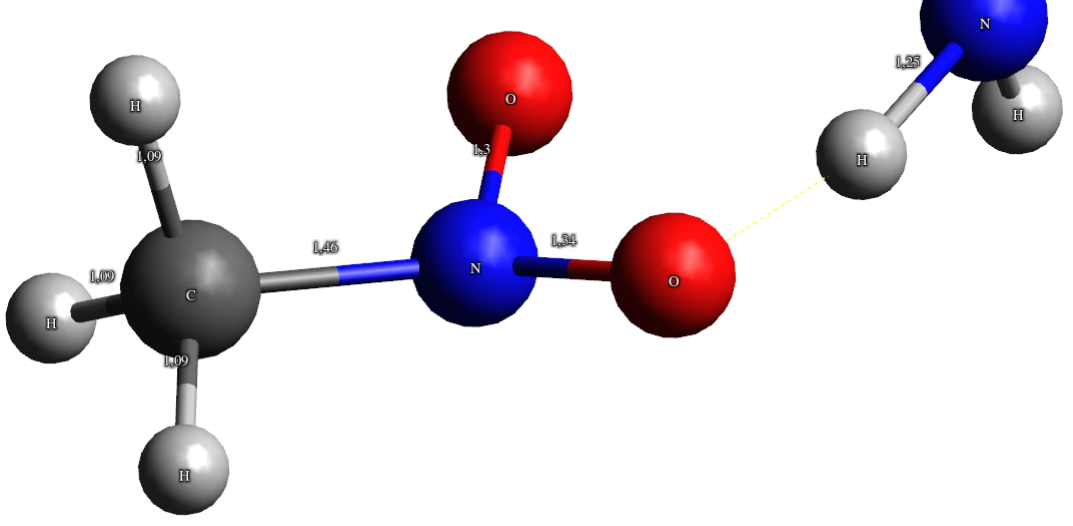

Figure 2: Transition state geometry of the reaction between ammonia and nitromethane

The TS of the reaction between ammonia and nitromethane using the AIM theory was examined. On the fig. 3 we can observe some critical points of the (3,-1)-type. The critical point number 21 is obviously for the TS, but the point number 20 is not. As you can see, this point connects the $\mathrm{O} 2$ and N5 atoms. The length of this bond is $2.35 \AA$. It can be considered as it is the hydrogen bond. Hence rupture of the bonds connecting the post-complex demands more energy than hydrogen abstraction. The point number 19 (type $(3,+1))$ spawns to satisfy the Poincare-Hopf rule [12]:

atom poins - bond points + ring points - cage points $=1$

$11-11+1-0=1$ 


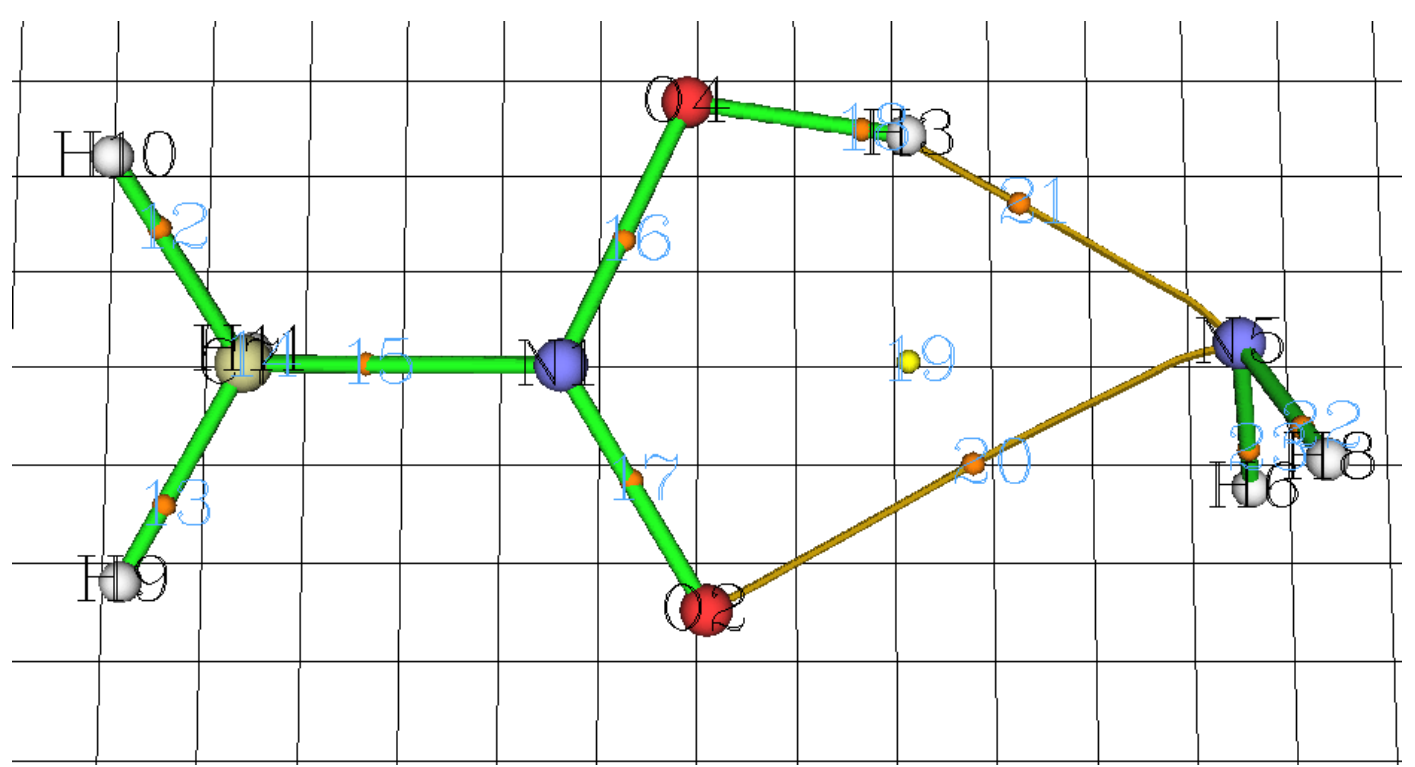

Figure 3: Topology of the TS between ammonia and triplet nitromethane. Orange balls are the $(3,-1)$ critical poins, yellow ball is the $(3,+1)$-type point

The second reaction is the methylamine and triplet nitromethane interaction. The mechanism is the same as. Due to the presence of methyl group contrary to the ammonia molecule, we can expect drop of the activation energy. So the ZPE-corrected activation barrier is of $4.9 \mathrm{~kJ} \cdot \mathrm{mol}^{-1}\left(1.2 \mathrm{kcal} \cdot \mathrm{mol}^{-}\right.$ $\left.{ }^{1}\right)$. The difference between this energy and an activation energy of the previous reaction is $8.4 \mathrm{~kJ} \cdot \mathrm{mol}^{-1}$.

On the fig. 4 you can see the Localized Orbital Locator (LOL) spread for the TS between monomethylamine and nitromethane. Violet color means no LOL on the current point and white color means that LOL value is greater than 0.8. Blue contour line denotes Van-der-Waals radius of the system. $\mathrm{N}$ label on the top of the picture is nitrogen atom of monomethylamine. Consequently, $\mathrm{N}$ label below denotes nitrogen atom of the $\mathrm{NO}_{2}$ group. Between left oxygen atom and upper nitrogen atom you can see the hydrogen atom proceeding to the nitrocompound from the $\mathrm{NH}_{2}$ group. Now take a look at the right-side oxygen atom. Slightly decreased LOL areas near the atom denotes electron pairs, are seen on the nitrogen and two pairs on the oxygen. One of the electron pairs on the right oxygen interacts with the nitrogen atom of the methylamine. The nature of that bonding we can guess as donor-acceptor.

The reaction between phenylamine (aniline) and triplet nitromethane was investigated. The mechanism of this process is the same as hydrogen abstraction by nitromethane. The expected plain geometry of the aniline molecule is not present; $\mathrm{NH}_{2}$ group lays in the other plane than benzene ring.

The activation energy of the reaction is of $6.3 \mathrm{~kJ} \cdot \mathrm{mol}^{-1}\left(1,51 \mathrm{kcal} \cdot \mathrm{mol}^{-1}\right)$. As expected, this value is less than energy of the reaction with ammonia, but greater than with the monomethylamine. 


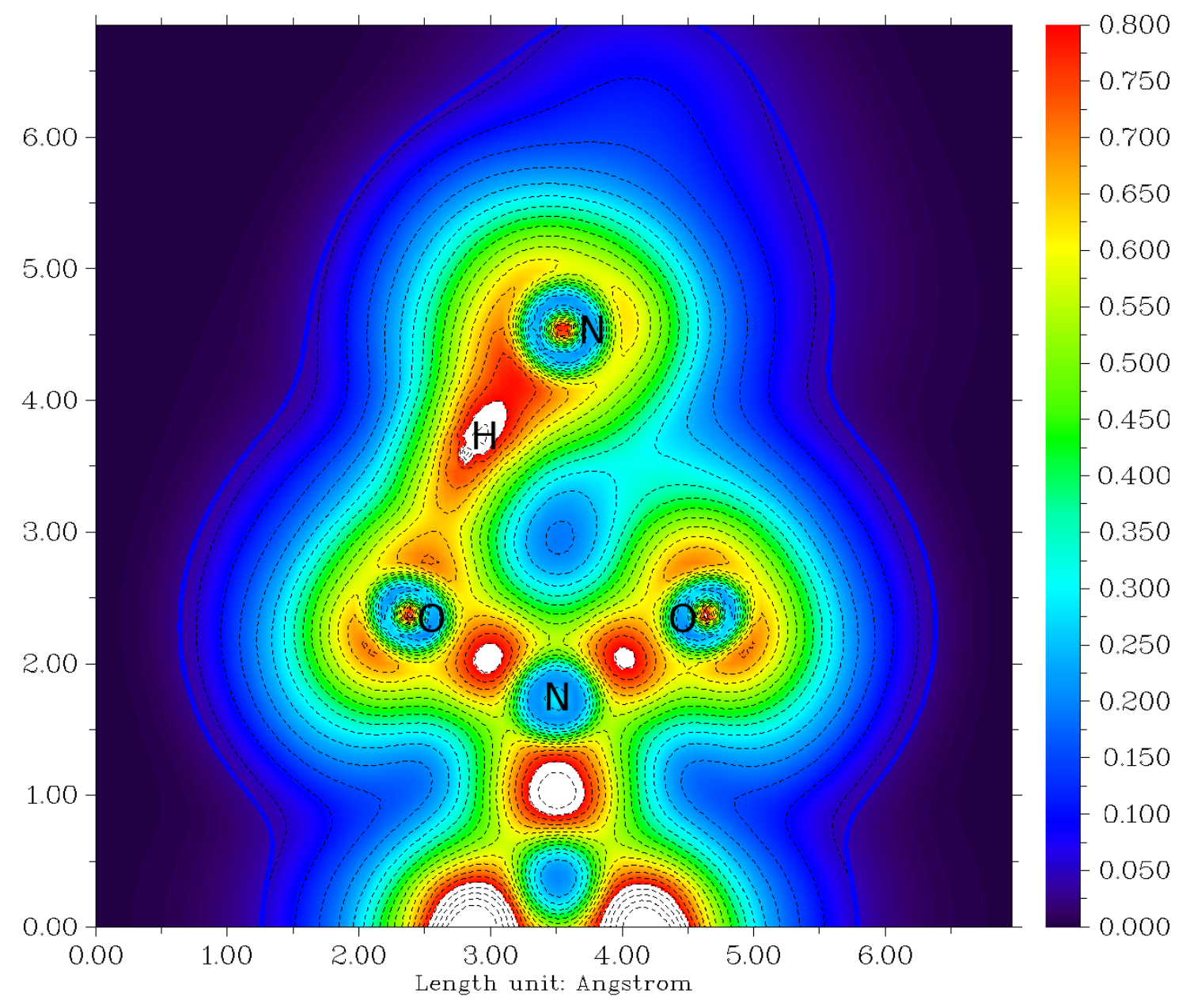

Figure 4: Localized Orbital Locator of the TS between monomethylamine and triplet nitromethane

\section{Conclusions}

Some reactions between excited nitromethane and amines were investigated using DFT method calculations. The predicted values for the activation energies are extremely low. But the strong coupling between nitrogen and oxygen atoms was found. It would be the more strong barrier for the next step of the reaction. The resulting reactions are

\begin{tabular}{|c|c|}
\hline $\mathrm{CH}_{3} \mathrm{NO}_{2}+\mathrm{NH}_{3} \rightarrow \mathrm{CH}_{3} \mathrm{NO}_{2} \mathrm{H} \cdot+\cdot \mathrm{NH}_{2}$ & $\mathrm{E}_{\mathrm{a}}=13.3 \mathrm{~kJ} \cdot \mathrm{mol}^{-1}$ \\
\hline $\mathrm{CH}_{3} \mathrm{NO}_{2}+\mathrm{NH}_{2} \mathrm{CH}_{3} \rightarrow \mathrm{CH}_{3} \mathrm{NO}_{2} \mathrm{H} \cdot+\cdot \mathrm{NHCH}_{3}$ & $\mathrm{E}_{\mathrm{a}}=4.9 \mathrm{~kJ} \cdot \mathrm{mol}^{-1}$ \\
\hline $\mathrm{CH}_{3} \mathrm{NO}_{2}+\mathrm{NH}_{2} \mathrm{Ph} \rightarrow \mathrm{CH}_{3} \mathrm{NO}_{2} \mathrm{H} \cdot+\cdot \mathrm{NHPh}$ & $\mathrm{E}_{\mathrm{a}}=6.3 \mathrm{~kJ} \cdot \mathrm{mol}^{-1}$ \\
\hline
\end{tabular}




\section{Acknowledgments}

Authors thanks Volga Research and Educational Center for Supercomputer Technologies for granting access to the computational resources on the cluster "Lobachevsky".

\section{Abbreviations}

PES Potential Energy Surface

EOM-CCSD Equation Of Motion Coupled Clusters Singles and Doubles

DFT Density Functional Theory

TS Transition State

ZPE Zero Point Energy

NEB Nudged Elastic Band

AIM Atoms In Molecules

LOL Localized Orbital Locator

\section{References}

[1] M. Valiev et al., "NWChem: A comprehensive and scalable open-source solution for large scale molecular simulations," Comput. Phys. Commun., vol. 181, no. 9, pp. 1477-1489, Sep. 2010.

[2] T. D. Crawford and H. F. Schaefer, "An Introduction to Coupled Cluster Theory for Computational Chemists," in Reviews in Computational Chemistry, K. B. Lipkowitz and D. B. Boyd, Eds. John Wiley \& Sons, Inc., 2000, pp. 33-136.

[3] R. M. Parrish et al., "Psi4 1.1: An Open-Source Electronic Structure Program Emphasizing Automation, Advanced Libraries, and Interoperability," J. Chem. Theory Comput., vol. 13, no. 7, pp. 3185-3197, Jul. 2017.

[4] Avogadro: an open-source molecular builder and visualization tool. Version 1.2.0. http://avogadro.cc/. .

[5] M. D. Hanwell, D. E. Curtis, D. C. Lonie, T. Vandermeersch, E. Zurek, and G. R. Hutchison, "Avogadro: an advanced semantic chemical editor, visualization, and analysis platform," $J$. Cheminformatics, vol. 4, p. 17, Aug. 2012.

[6] N. M. O’Boyle, M. Banck, C. A. James, C. Morley, T. Vandermeersch, and G. R. Hutchison, "Open Babel: An open chemical toolbox," J. Cheminformatics, vol. 3, p. 33, Oct. 2011.

[7] T. Lu and F. Chen, "Multiwfn: A multifunctional wavefunction analyzer," J. Comput. Chem., vol. 33, no. 5, pp. 580-592, Feb. 2012.

[8] T. Williams, C. Kelley, and and others, Gnuplot 5.2: an interactive plotting program. .

[9] A. K. Chandra, "Origin of the activation barrier in the process of hydrogen abstraction: the perturbation treatment," J. Photochem., vol. 18, no. 2, pp. 151-159, Jan. 1982.

[10] N. C. de Lucas et al., "A combined laser flash photolysis, density functional theory and atoms in molecules study of the photochemical hydrogen abstraction by pyrene-4,5-dione," J. Photochem. Photobiol. Chem., vol. 201, no. 1, pp. 1-7, Jan. 2009.

[11] D. Ovsyannikov, D. Fomichev, and S. Zelentsov, "Teoretical study of photooxidation of ammonia by nitromethane," 01-Nov-2016. [Online]. Available: http://sciforum.net/conference/ecsoc20/paper/3543. [Accessed: 24-Oct-2017].

[12] R. F. W. Bader, Atoms in Molecules: A Quantum Theory. Oxford, New York: Oxford University Press, 1994. 